Article

\title{
Theoretical Model and Numerical Analysis for Asymmetry of Shock Train in Supersonic Flows
}

\author{
Bo Sun ${ }^{1, *}$, Chengpeng Wang ${ }^{2}$ and Changfei Zhuo ${ }^{1}$ \\ 1 Department of Aerospace Engineering, Nanjing University of Science and Technology, Nanjing 210094, \\ China; njustzcf203@126.com \\ 2 College of Aerospace Engineering, Nanjing University of Aeronautics and Astronautics, Nanjing 210016, \\ China; wangcp@nuaa.edu.cn \\ * Correspondence: hypersun@njust.edu.cn
}

Received: 5 February 2020; Accepted: 4 March 2020; Published: 2 April 2020

check for updates

\begin{abstract}
The reason for the asymmetry phenomenon of Shock/Boundary Layer Interactions (SBLI) in a completely symmetric duct with symmetric flow conditions is still an open question. A theoretical model for the asymmetry of shock train in supersonic flows is proposed based on the properties of fluid entrainment in the mixing layer and momentum conservation. Flow deflection angles downstream from different SBLI regions were deduced from this model. Steady numerical simulations were conducted to model the asymmetry of the SBLI in an isolator tested by Carroll. The obtained deflection angles using the theoretical model with the aerodynamic parameters from the numerical results of forced symmetric isolator flow give a larger range estimation and a close average value compared with the asymmetric full duct flow. This showed that the entrainment of shear layer on the separation induced by SBLI plays a dominant role in the asymmetry of the confined SBLI.
\end{abstract}

Keywords: asymmetry; shock train; Coanda effect; isolator; supersonic flow

\section{Introduction}

The asymmetry phenomenon of Shock/Boundary Layer Interactions (SBLI) in a completely symmetric duct with symmetric flow conditions, e.g., an asymmetric shock train (Figure 1), has been observed frequently by many researchers. The reason for the flow asymmetry is still an open question, and has been clarified neither by experiment nor by numerical simulation [1].

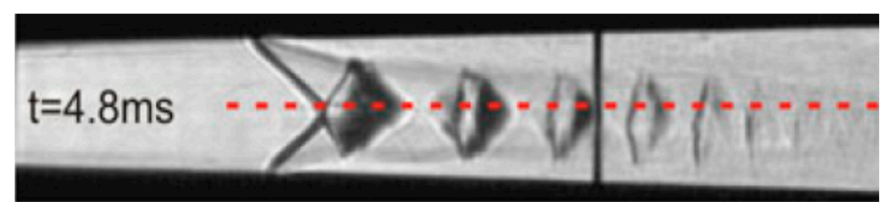

Figure 1. Asymmetric shock train in a nozzle with a small divergence angle [2].

The asymmetric shock system in a symmetric and nearly constant area rectangular duct was discovered experimentally by many researchers. Ikui et al. [3] investigated shock trains at Mach numbers 1.33 to 2.79 and reported that when the airstream Mach exceeded 1.60 considerable flow asymmetry was observed. The asymmetry of shock train was described in detail in the series of experiments at Ma2.45 carried out by Carroll and Dutton [4,5]. They found that there are two modes of unsteadiness in the oblique shock train: a high-frequency streamwise oscillation and a low-frequency transverse oscillation related to the neutrally stable, asymmetric character of the shock pattern, i.e., the shock system is sometimes attached to the lower wall and is sometimes attached to the upper wall, flipping from one wall to the other during the course of a test run. In addition, the frequency 
of second flipping oscillation rises as the confinement parameter $\delta_{u} / h$ increases. Their experiments at three different confinement levels showed that the shock train is more asymmetric at $\delta_{u} / h=0.26$ than those at 0.15 and 0.35 . Sugiyama et al. [6,7] observed an asymmetric oblique shock train in Mach 4.0 experiments. Gawehn et al. [2] also observed the flipping unsteadiness of a shock train in a Laval nozzle with a small divergence angle.

The reason for the asymmetry of confined SBLI is not clear yet, but many researchers, e.g., Lawrence [8], Papamoschou and Zill [9], Myshenkov [10], and Wang [11], attributed it to the Coanda effect, which is used for the tendency of a fluid jet issuing tangentially onto a curved or angled solid surface to adhere to it $[12,13]$. The entrainment of the jet on ambient fluid is regarded as the cause for Coanda effect which has been applied widely in industry. The Coanda effect was confirmed experimentally by Allery et al. [14] to work in an asymmetrical configuration as it does in a single wall case: the jet reattaches randomly to either of two walls. Wang et al. [15] created a theoretical model for asymmetry of SBLI in nozzle flows based on the properties of fluid entrainment in the mixing layer. Verma et al. [16] conducted experiments on Ma2.0 planar nozzles, with results indicating that the state of the boundary layer and the separated shear layer to the nozzle walls seem to play a dominant role in initiating conditions that favor the origin of flow asymmetry in nozzles. Telega et al. [17] experimentally found that if the sizes of $\lambda$-feet are measured in ratio to the height of division point, both their normalized sizes are the same.

This study was motivated by the research work of Piponniau et al. [18] who proposed a model based on the properties of fluid entrainment in the mixing layer to explain low frequency unsteadiness on shock induced separation. Due to the close relation between Piponniau's model and the Coanda effect, his model is developed further in this paper to create a theoretical model for the asymmetry of shock train in a nearly constant area duct whose flowfield data were obtained by numerical simulations. The shock train flow in a full duct tested by Carroll [5] was simulated numerically for the numerical method verification and for comparison with the theoretical results.

\section{Theoretical Considerations}

\subsection{Aerodynamic Scheme}

According to Piponniau et al. [18], when a separation bubble is produced by SBLI (Figure 2), eddies form from the separation line in the mixing layer zone (the reversed flow in the separation bubble is regarded as the other stream) and grow as the separation bubble moves downstream. Fluid from the separation zone is entrained by the mixing layer. Near the middle of the bubble (where the mixing layer has the maximum thickness), these eddies shed into the downstream flow, bringing with them their mass, momentum, and vorticity outside the separation region. This generates a default of mass in the separation region, which increases over time. Therefore, when the flow reattaches downstream, the mass amount inside the bubble decreases.

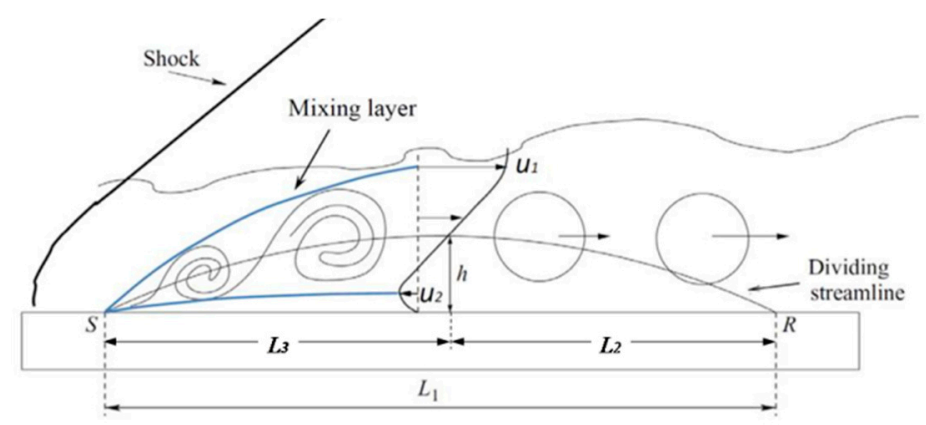

Figure 2. Sketch of the entrainment of mixing layer on the separation bubble [18].

Now, we consider the situation of an asymmetric SBLI in a 2D duct, whose flowfield pattern is sketched in Figure 3. The Coanda effect works in this flow, and the entrainment of mixing layer 
on a separation bubble near the lower wall reduces the mass and pressure inside the bubble, and the opposite phenomena happen near the upper wall. The pressure difference between two sides deflects the shock train to the lower wall. To understand how the result of asymmetry occurs, an imaginary symmetric flowfield, which is supposed to be the situation before the asymmetric flow pattern appearing, is sketched in Figure 4. Prior to creating the asymmetry model, three assumptions are made in this situation:

(1) Based on the features of the Coanda effect introduced in Section 1, it is assumed that the entrainment of mixing layer only activates on one side, while the separation bubble on the other side stays fixed.

(2) After the shedding of eddies, the mass and pressure are reduced inside the bubble but its size stays constant.

(3) If the final flow is still symmetric, a new amount of reversed flow from downstream enters the bubble at reattachment point $R$ to ensure the balance [19].

In the plane reflected shock case, large vortices are shed downstream at the position where the mixing layer reaches its maximum thickness, i.e., the position with maximum separation bubble thickness, which is approximately at the middle of separation bubble [20]. The distance from the shedding position of large vortices to the reattachment point is denoted as $L_{2}$ (Figure 2).

Based on the above assumptions and deduction, we now discuss the total mass entrained from separation bubble. Total entrained mass is the product of entrainment mass rate $\dot{m}_{e}$ and entrainment time $T_{e}$. The rate of mass entrainment can be obtained from Piponniau's model (will be deduced later). According to the third assumption, the distance between mass escaping from the bubble and mass returning into the bubble is $L_{2}$, and then the time that can be used for entrainment is given by:

$$
T_{e}=\frac{L_{2}}{a_{2}}
$$

where $a_{2}$ is the sonic speed on the low-velocity side of the mixing layer.

With the total entrained mass, the pressure drop inside the bubble after the entrainment can be obtained based on the second assumption. For the shock train case, a remarkable difference in the shock patterns between Figures 2 and 4 is that there are multiple SBLIs. Therefore, the above model for the single SBLI was applied to each SBLI in shock train. The total force difference between two sides of the duct was obtained by integrating pressure drops of all SBLIs. Finally, the deflection angle downstream the shock train was obtained from the longitudinal momentum conservation equation, which was compared with the actual deflection angle of the flow.

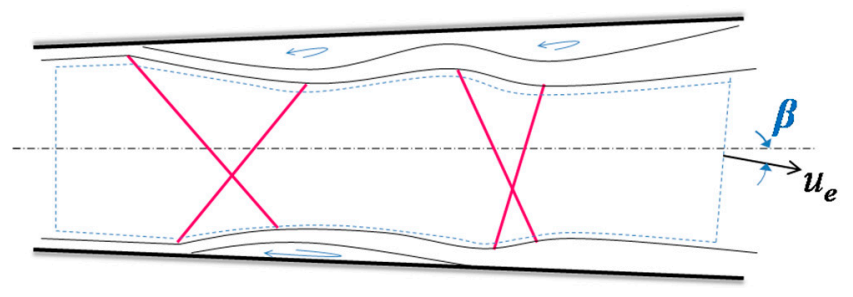

Figure 3. Asymmetric multiple Shock/Boundary Layer Interactions (SBLI) pattern in a 2D duct. 


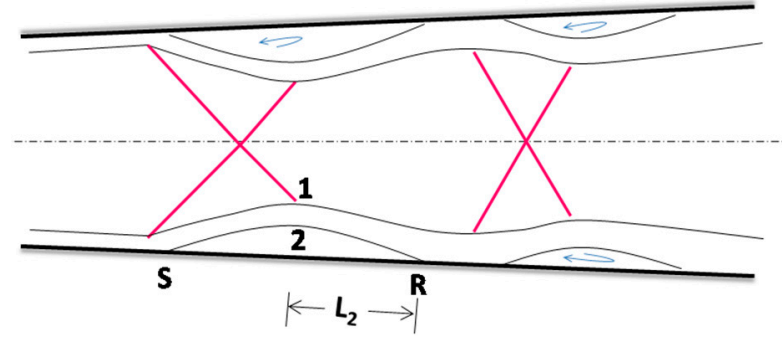

Figure 4. Imaginary symmetric SBLI flowfield before asymmetric flow taking shape in a 2D duct.

\subsection{Theoretical Model for Flow Deflection with Entrainment for Multiple SBLIs}

According to Piponniau's entrainment model, the separation bubble is approximated by a triangle of length $L_{1}$ and height $h$ (Figure 2) with an average density of $\rho_{m}$. Then, the air mass in the bubble by unit span is given by:

$$
M_{b}=\frac{1}{2} \rho_{m} L_{1} h .
$$

The rate of mass entrainment [18] is:

$$
\dot{m}_{e}=\int_{\delta_{2}\left(x_{0}\right)}^{y_{0}\left(x_{0}\right)} \rho u d y=\rho_{m} \delta_{w}\left(x_{0}\right) \int_{-\frac{1}{2}}^{0} u d \eta=\rho_{m} u_{1} \delta^{\prime}{ }_{w} x_{0}\left[(1-r) C+\frac{r}{2}\right]
$$

where $\delta_{2}(x)$ is the vertical coordinate of the edge of the mixing layer on the low-velocity side and $\mathrm{y}_{0}(\mathrm{x})$ is the vertical coordinate of the centerline of the mixing layer, and $x_{0}=L_{3}=L_{1}-L_{2}$ denotes the position where large eddies shed, $\eta$ is the similarity variable, the constant $C \cong 0.14, \delta_{w}$ and $\delta_{w}{ }^{\prime}$ are respectively the local thickness and the spreading rate of the mixing layer, and the latter can be expressed by the following relation according to Papamoschou and Roshko [21]:

$$
\delta^{\prime}{ }_{w}=\frac{\delta^{\prime}{ }_{r e f}}{2} \frac{(1-r)(1+\sqrt{s})}{1+r \sqrt{s}} \Phi\left(M_{c}\right)
$$

where $\delta_{\text {ref }} \cong 0.16$ is the spreading rate for subsonic half jet [22]. $r=u_{2} / u_{1}$ and $s=\rho_{2} / \rho_{1}$ are velocity ratio and density ratio across the mixing layer, respectively. The function $\Phi\left(\mathrm{M}_{\mathrm{c}}\right)$ is the normalized spreading rate of mixing layer and is dependent on the convective Mach number $M_{c}$ :

$$
M_{c}=\frac{u_{1}-u_{2}}{a_{1}+a_{2}}
$$

$\Phi\left(M_{c}\right)$ must be determined by experiment [23] and Figure 5 gives its empirical value depending on the convective Mach number. Piponniau et al. [18] introduced a function $g(r, s)$ as:

$$
g(r, s)=\frac{\delta^{\prime} r e f}{2} \frac{(1-r)(1+\sqrt{s})}{1+r \sqrt{s}}\left[(1-r) C+\frac{r}{2}\right]
$$

Finally, we obtain the rate of mass entrainment:

$$
\dot{m}_{e}=\rho_{m} L_{3} u_{1} \Phi\left(M_{c}\right) g(r, s)
$$




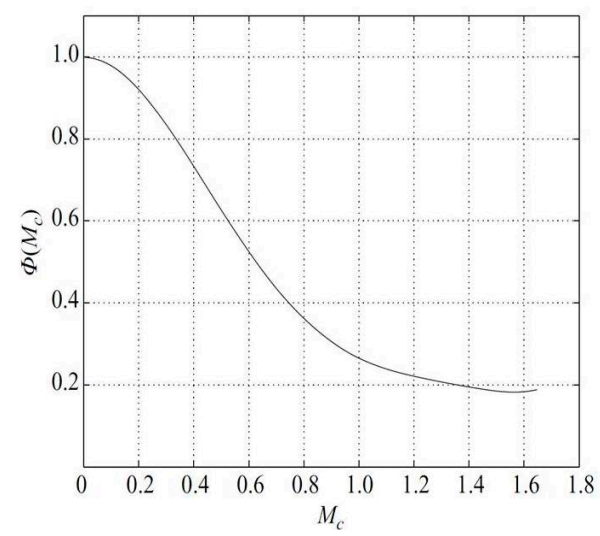

Figure 5. Normalized spreading rate as a function of the convective Mach number [18].

Consequently, we discuss the pressure variation in the bubble. The total mass of entrainment is:

$$
M_{e}=\dot{m}_{e} T=\rho_{m} L_{3} L_{2} \frac{u_{1}}{a_{2}} \Phi\left(M_{c}\right) g(r, s)
$$

Based on the second assumption, the volume of bubble keeps constant, and the temperature in the bubble is considered as invariant, then the density and pressure in the bubble after entraining are:

$$
\begin{gathered}
\rho_{e}=\frac{M_{b}-M_{e}}{V_{b}}=\rho_{m}\left[1-\frac{2 L_{3} L_{2} u_{1}}{L_{1} h a_{2}} \Phi\left(M_{c}\right) g(r, s)\right] \\
p_{e}=\frac{\rho_{e}}{\rho_{m}} p_{m}=p_{m}\left[1-\frac{2 L_{3} L_{2} u_{1}}{L_{1} h a_{2}} \Phi\left(M_{c}\right) g(r, s)\right]
\end{gathered}
$$

where $p_{m}$ is the initial average pressure in the bubble. Then, according to the first assumption, we can obtain the pressure drop in the bubble:

$$
\left.\Delta p=\frac{2 L_{3} L_{2} u_{1}}{L_{1} h a_{2}} p_{m} \Phi\left(M_{c}\right) g(r, s)\right]
$$

Equation (11) can be written as:

$$
\Delta p=\frac{2 L_{3} L_{2}}{L_{1} h} M_{1} \sqrt{\frac{T_{1}}{T_{2}}} p_{2} \Phi\left(M_{c}\right) g(r, s)
$$

$p_{m}=p_{2}$ is applied in Equation (12) where $p_{2}$ denotes the static pressure near the point 2 in Figure 4.

Equation (12) is applied to each SBLI of shock train (Figure 4) to obtain the pressure drop in each divided separation bubble. Then, based on the pressure drops in all bubbles, we calculate the deflection angle of flow. Writing the vertical momentum conservation for the control volume in Figure 3, we obtain:

$$
\sum_{1}^{n} \Delta p_{i} L_{1 i}-p_{e} h_{e} \sin \beta=\dot{m} u_{e} \sin \beta
$$

where $n$ is the number of divided separation bubble in shock train, $L_{1 i}$ is the length of each divided separation bubble, $p_{e}$ is the pressure at the end of control volume, $h_{e}$ is the duct height at the end of control volume, $\dot{m}$ is the mass flow rate of the main stream, $u_{e}$ is the main stream velocity at the exit, and $\beta$ is the flow deflection angle from horizontal direction (Figure 3). Then from Equation (13), we obtain:

$$
\beta=\arcsin \left(\frac{\sum_{1}^{n} \Delta p_{i} L_{1 i}}{p_{e} h_{e}+\dot{m} u_{e}}\right)
$$




\section{Model Application Based on Numerical Results of An Isolator}

\subsection{Isolator Geometry and Numerical Methods}

Carroll's shock train experiments at Ma2.45 [5] were chosen to verify the model for asymmetry proposed in Section 2. The experiments were conducted in a planar, two-dimensional supersonic wind tunnel with a nozzle exit height of $38.1 \mathrm{~mm}$ and a test section length of $754 \mathrm{~mm}$. Both top and bottom walls have a divergence angle of $0.25^{\circ}$. Simulations were not attempted to model the whole test duct but started from the onset of SBLI where the duct has a height of $40.2 \mathrm{~mm}$. The inlet conditions were as follows: Mach number $M_{0}=2.45$, total pressure $p_{t 0}=310 \mathrm{kPa}$, total temperature $T_{t 0}=295 \mathrm{~K}$, and the inlet boundary layer velocity profile was given by a $1 / 7$ power-law with a boundary layer thickness of $5.4 \mathrm{~mm}$. The simulations for both full and forced symmetric ducts were conducted.

The Reynolds-averaged governing equations for compressible turbulent flow with a two-equation SST (Shear Stress Transport) turbulence model in the CFX software were employed to simulate idea gas $(\gamma=1.4)$ steady flows. The computational domain and boundary conditions of these two models are shown in Figure 6. Two kinds of meshes were used for the full nozzle simulations to judge the mesh sensitivity: the coarse one had 89,800 grids and the fine one had 195,247 grids. The total number of grid used was 51,900 for the half duct. All meshes used in the simulations had a higher density near the wall and the minimum first grid point from the walls provided a $y^{+}<1$. An adiabatic, no slip wall boundary condition was specified for all the walls in these simulations. Back pressure with a ratio of 5.85 relative to the inlet static pressure was imposed on the outlet boundary to produce the shock train in the ducts.

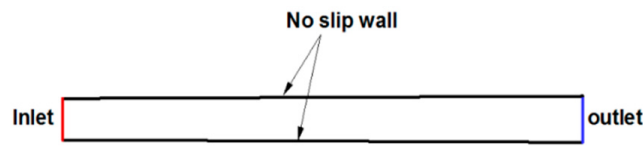

(a)

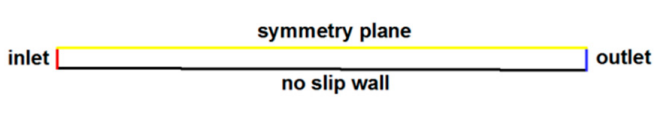

(b)

Figure 6. Computational domain and boundary conditions for isolator flow simulations: (a) Full isolator; (b) forced symmetric half isolator.

\subsection{Numerical Verification of Asymmetric SBLI in Full Duct}

An asymmetric oblique shock train attached to the bottom wall was obtained from the simulation with the coarse mesh for the full duct (Figure 7a), which is similar to the experimental Schlieren picture in Figure 7c from Carroll [5]. The oblique shock train consists of three pairs of distinct shock waves and other weaker shock waves. The angle between the centerline of the shock train and the centerline of the duct was around $3.9^{\circ}$, which represents the maximum deflection in the shock train region. Figure 7a shows a large closed separation bubble near the top wall that is longer than the shock train. On the bottom wall, there is a small obvious separation bubble caused by the first SBLI. An asymmetric oblique shock train attached to the top wall (Figure $7 \mathrm{~b}$ ) was observed from the simulation with the fine mesh; however, except for this difference, the other flowfield features including the shock train deflection angle were similar as the flowfield in Figure 7a. The difference of asymmetry direction of the shock train is caused by its neutral stability which was observed in the experiments by Carroll [5], i.e., the shock system sometimes is attached to the lower wall and sometimes is attached to the upper wall, flipping from one wall to the other during the course of a test run. 


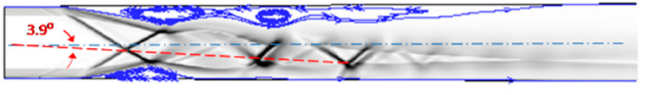

(a)

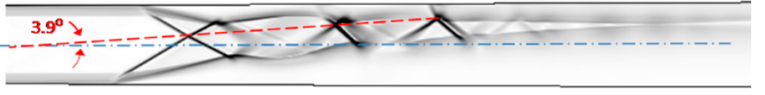

(b)

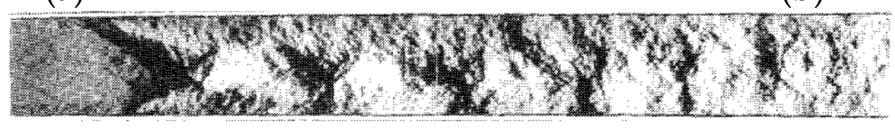

(c)

Figure 7. Schlieren picture of shock train from simulation and experiment: (a) Simulation with the coarse mesh; (b) simulation with the fine mesh; (c) experiment [5].

Wall pressure distributions from simulation with the coarse mesh also agreed well with experimental results (Figure 8), and both results showed an obvious asymmetry of shock train. The pressure vibration along the bottom wall was caused by the shock train, while there was no distinct pressure vibration along the top wall due to the shock train being isolated by the large separation bubble near the wall.

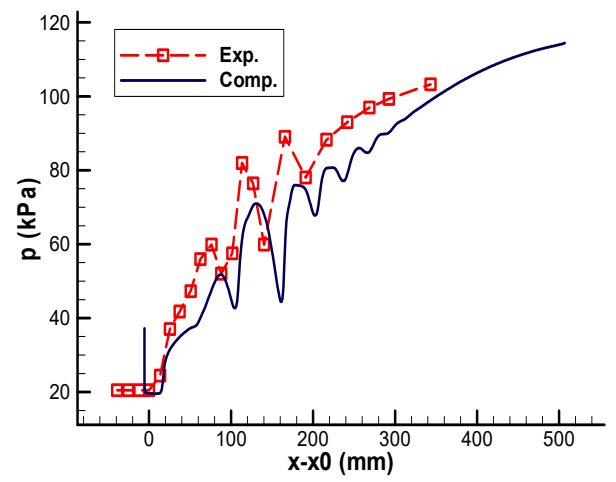

(a)

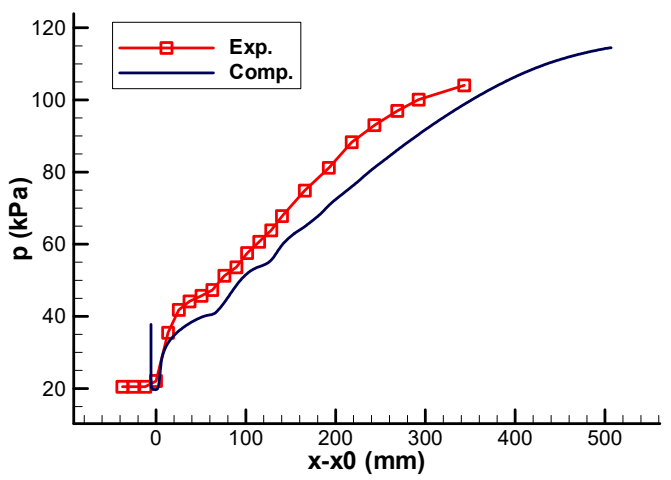

(b)

Figure 8. Wall pressure distributions from simulation and experiment $\left(x_{0}\right.$ denotes the onset of the SBLI): (a) bottom wall; (b) top wall.

\subsection{The Overall Deflection Angle Calculation for Asymmetric Shock Train}

In order to evaluate the flow asymmetry in the whole shock train region, an overall deflection angle was defined by considering the streamtube deformation of the main stream. Three different streamtubes based on the main stream streamlines are shown in Figure 9, where the streamtube $A B_{1} C D_{1}$ covers the region of the first SBLI and the streamtube $A_{3} C D_{3}$ covers all the three SBLIs. The deflection angles of the upper side and the lower side of a streamtube were averaged to be taken as the overall deflection angle. Taking the streamtube $\mathrm{AB}_{3} \mathrm{CD}_{3}$ as an example, the angle between its upper side $\mathrm{AB}_{3}$ and the horizontal direction is $\theta_{3}$ (taking the counterclockwise as the positive), and the angle between its lower side $\mathrm{CD}_{3}$ and the horizontal direction is $\delta_{3}$, as shown in Figure 9. We suppose that for the symmetric shock train, the angle between its lower side and the horizontal direction is $\eta$ (here, $\eta$ is an absolute value), and the angle between its upper side and the horizontal direction is $-\eta$. Then, the deflection angle of the upper side $\mathrm{AB}_{3}$ from the imaginary symmetric flow to the actual asymmetric flow is:

$$
\alpha_{\text {upper }}=\theta_{3}-(-\eta)
$$

The deflection angle of the lower side $\mathrm{CD}_{3}$ is:

$$
\alpha_{\text {lower }}=\delta_{3}-\eta
$$


Finally, the overall deflection angle of the streamtube is calculated as:

$$
\beta_{3}=\frac{\alpha_{\text {lower }}+\alpha_{\text {upper }}}{2}=\frac{\delta_{3}+\theta_{3}}{2}
$$

The overall deflection angles of the three streamtubes in Figure 9 were obtained using Equation (17) and are shown in Figure 10 (the black solid line), where the deflection angle varies with a small range of $-2.6^{\circ}$ to $-2.1^{\circ}$ for the three different SBLI regions.

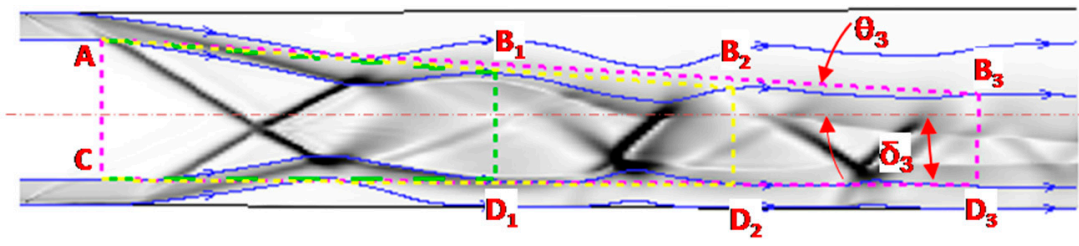

Figure 9. Three streamtubes including different SBLI regions for deflection angle calculations.

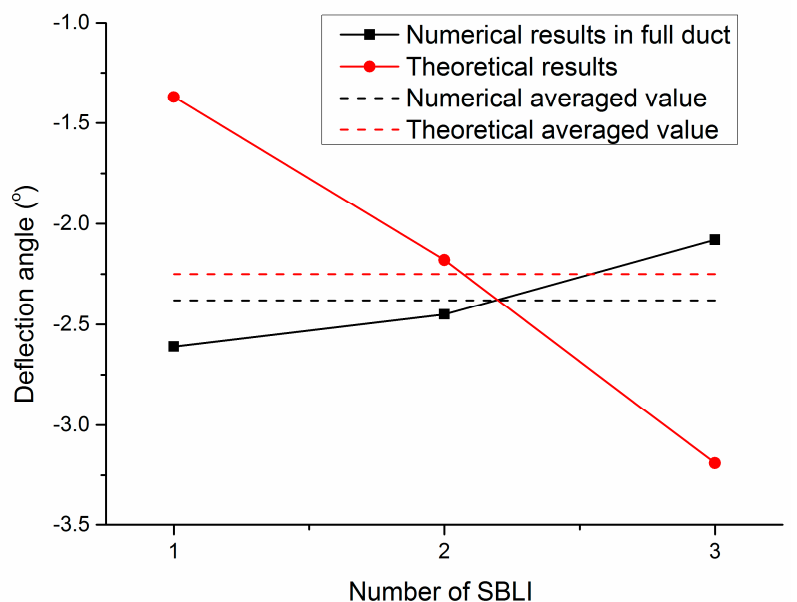

Figure 10. Streamtube deflection angles for three different SBLI regions from numerical and theoretical results.

\subsection{Model Application Based on Numerical Results of Half Duct with Forced Symmetry at Ma2.45}

The flowfield in the forced symmetric model in Figure $6 \mathrm{~b}$ was computed in order to apply the asymmetry model described in Section 2.2. The numerical results showed that a symmetric oblique shock train is produced and there are three obvious and almost independent separation bubbles caused by the first three strong SBLIs (Figure 11). Compared to the asymmetric shock train in the full duct, the pseudo-shock region in the half duct is shorter than that in the full duct, since the pressure increases more quickly in the half duct (Figure 12a). In Figure 12b, near the wall of the half duct, there are three distinct separation bubbles where the wall shear stress is lower than zero, as shown in Figure 11, although the first two bubbles are not uncoupled completely.

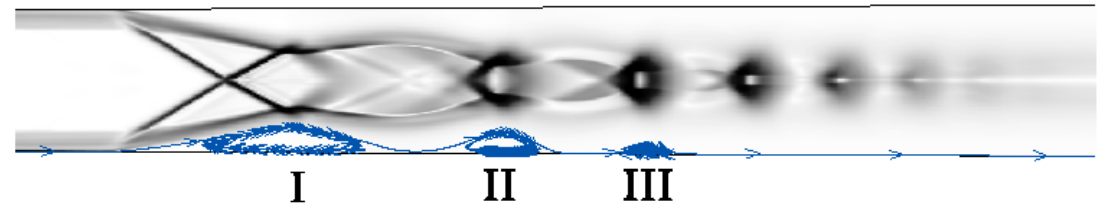

Figure 11. Numerical schlieren of shock train with forced symmetry (three separation bubbles caused by the first three SBLIs are labeled). 


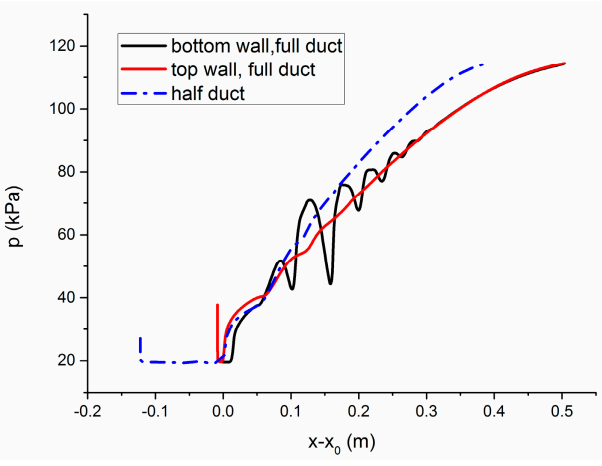

(a)

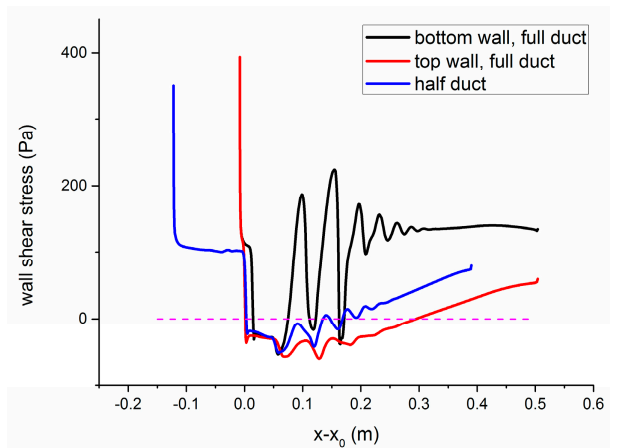

(b)

Figure 12. Wall pressure distributions and wall shear stresses for the full duct and the half duct $\left(x_{0}\right.$ denotes the onset of the SBLI): (a) Wall static pressure distribution; (b) wall shear stress.

The first SBLI had the largest separation bubble among the three SBLIs, whose velocity contours in the $x$ direction and streamlines are shown in Figure 13, and some necessary parameters of the bubble for the asymmetry model application described in Section 2.2 can be extracted from this figure and Figure 12b. The first bubble has a length of $87.2 \mathrm{~mm}$ and a height of $7.69 \mathrm{~mm}$. The main stream velocity in the $\mathrm{x}$ direction near the highest point of the mixing layer is taken as the high-velocity side velocity of the mixing layer, i.e., $u_{1}$ is $469 \mathrm{~m} / \mathrm{s}$ for the first SBLI. The low-velocity side velocity is taken from the maximum reversed velocity in the separation bubble, i.e., $u_{2}$ is $-76 \mathrm{~m} / \mathrm{s}$ in Figure 13. After obtaining all the necessary aerodynamic parameters of the first separation (Table 1), Equation (14) was applied to calculate the theoretical flow deflection angle downstream the first SBLI (now $n=1$ in Equation (14)).

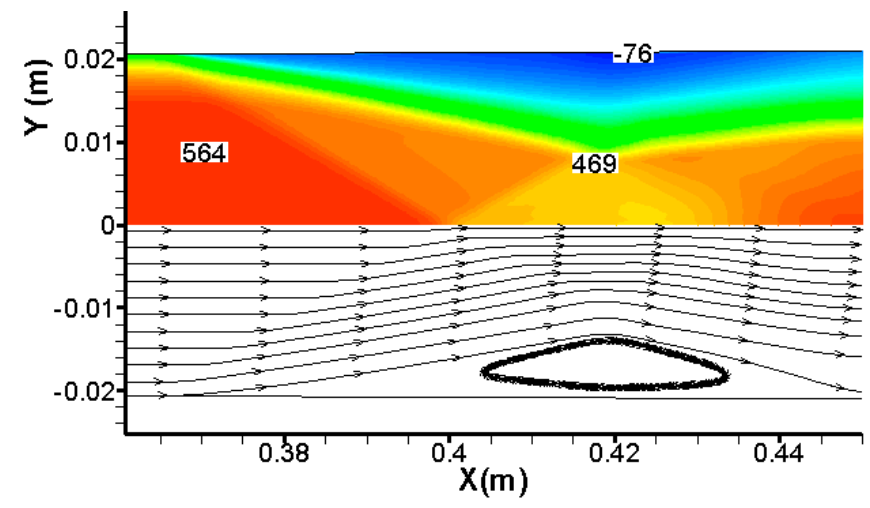

Figure 13. Velocity-x contours $(\mathrm{m} / \mathrm{s})$ and streamlines near the first separation bubble.

Table 1. Aerodynamic parameters of the separation and asymmetry for the first SBLI of shock train with forced symmetry.

\begin{tabular}{cccccccccccccccc}
\hline Part & $T_{1} / \mathbf{T}_{2}$ & $\begin{array}{c}u_{1} \\
(\mathbf{m} / \mathbf{s})\end{array}$ & $r$ & $s$ & $\begin{array}{c}g(r, s) \\
\times \mathbf{1 0}^{2}\end{array}$ & $M_{c}$ & $\mathbf{\Phi}\left(M_{c}\right)$ & $\begin{array}{c}h \\
(\mathbf{m m})\end{array}$ & $\begin{array}{c}L_{1} \\
(\mathbf{m m})\end{array}$ & $\begin{array}{c}L_{2} \\
(\mathbf{m m})\end{array}$ & $\begin{array}{c}\Delta \mathbf{p} \\
(\mathbf{P a})\end{array}$ & $p_{e} / p_{t 0}$ & $\begin{array}{c}h_{e} \\
(\mathbf{m m})\end{array}$ & $\dot{m} u_{e}(\mathbf{N})$ & $\begin{array}{c}\beta_{1} \\
\left({ }^{\circ}\right)\end{array}$ \\
\hline $\mathrm{I}$ & 0.64 & 469 & -0.162 & 0.391 & 1.35 & 0.88 & 0.32 & 7.69 & 87.2 & 35.0 & 1218 & 0.097 & 21 & 3927 & -1.4 \\
\hline
\end{tabular}

A similar process was also applied to the other two separation bubbles, and then the necessary parameters of these three separation bubbles were obtained for the calculation of the asymmetry model and are listed in Table 2. Finally, the theoretical deflection angles of the flow behind the second and the third SBLI were also calculated using Equation (14). These three theoretical deflection angles for the three different positions are shown in Figure 10 (the red solid line). Compared with the actual deflection angles from the full duct simulation, the theoretical result produced a larger angle variation, and the averaged deflection angles from the theoretical result and the actual full duct were very close (two dashed lines in Figure 10). 
Table 2. Aerodynamic parameters of the separation and asymmetry for the three SBLIs with forced symmetry.

\begin{tabular}{|c|c|c|c|c|c|c|c|c|c|c|c|c|c|c|c|}
\hline Part & $T_{1} / T_{2}$ & $\begin{array}{c}u_{1} \\
(\mathrm{~m} / \mathrm{s})\end{array}$ & $r$ & $s$ & $\begin{array}{l}g(r, s) \\
\times 10^{2}\end{array}$ & $M_{c}$ & $\Phi\left(M_{c}\right)$ & $\begin{array}{c}h \\
(\mathrm{~mm})\end{array}$ & $\begin{array}{c}L_{1} \\
(\mathrm{~mm})\end{array}$ & $\begin{array}{c}L_{2} \\
(\mathrm{~mm})\end{array}$ & $\begin{array}{c}\Delta p \\
(\mathbf{P a})\end{array}$ & $p_{e} / p_{t 0}$ & $\begin{array}{c}h_{e} \\
(\mathrm{~mm})\end{array}$ & $\dot{m} u_{e}(\mathrm{~N})$ & $\begin{array}{l}\beta_{3} \\
\left(^{\circ}\right)\end{array}$ \\
\hline I & 0.64 & 469 & -0.162 & 0.391 & 1.35 & 0.88 & 0.32 & 7.69 & 87.2 & 35.0 & 1218 & & & & \\
\hline II & 0.76 & 381 & -0.125 & 0.418 & 1.53 & 0.67 & 0.46 & 6.32 & 44.0 & 17.5 & 1539 & 0.176 & 20 & 3387 & -3.2 \\
\hline III & 0.84 & 331 & -0.037 & 0.432 & 1.78 & 0.52 & 0.60 & 2.28 & 21.3 & 8.9 & 3402 & & & & \\
\hline
\end{tabular}

\section{Conclusions}

A theoretical model for the asymmetry of shock train in the nearly constant area duct was proposed based on the properties of fluid entrainment in the mixing layer and momentum conservation. The deflection angles of different positions in a shock train were calculated using the theoretical model with the aerodynamic parameters of separation bubbles from the simulation result of a forced symmetric shock train in a half duct at Ma2.45. The full duct flow was also simulated numerically for comparison with theoretical results. The theoretical and numerical results showed that:

(1) The flowfield of an asymmetric shock train in a full duct can be simulated satisfactorily numerically, which agrees well with the experimental result.

(2) The neutral stability of shock train asymmetry was observed in the steady simulations, i.e., the shock train attached randomly to the lower wall or to the upper wall of the duct for the different simulation runs.

(3) The theoretical model for the shock train asymmetry produces a larger range estimation and a close averaged value of the deflection angle compared to the actual full duct flow.

Author Contributions: Conceptualization, B.S.; methodology, B.S. and C.W.; software, C.Z.; validation, C.W.; formal analysis, B.S.; investigation, B.S. and C.W.; data curation, C.Z.; writing-original draft preparation, B.S.; supervision, C.W. All authors have read and agreed to the published version of the manuscript.

Funding: This research received the support of the National Natural Science Foundation of China (Grant No. 11602109).

Acknowledgments: The first author wishes to thank Herbert Olivier at Shock wave laboratory, RWTH Aachen University for the helpful discussion with him.

Conflicts of Interest: The authors declare no conflict of interest.

\section{References}

1. Hadjadj, A.; Onofri, M. Nozzle flow separation. Shock Waves 2009, 19, 163-169. [CrossRef]

2. Gawehn, T.; Gülhan, A.; Al-Hasan, N.S.; Schnerr, G.H. Experimental and numerical analysis of the structure of pseudo-shock systems in laval nozzles with parallel side walls. Shock Waves 2010, 20, 297-306. [CrossRef]

3. Ikui, T.; Matsuo, K.; Nagai, M.; Honjo, M. Oscillation Phenomena of Pseudo-Shock Waves. Bull. JSME 1974, 17, 1278-1285. [CrossRef]

4. Carroll, B.F.; Dutton, J.C. A Numerical and Experimental Investigation of Multiple Shock Wave/Turbulent Boundary Layer Interactions in a Rectangular Duct; Rept. UILU-ENG-88-4015; Department of Mechanical and Industrial Engineering, University of Illinois at Urbana-Champaign: Urbana, IL, USA, 1988.

5. Carroll, B.F.; Dutton, J.C. Characteristics of Multiple Shock Wave/Turbulent Boundary-Layer Interactions in Rectangular Ducts. J. Propuls. Power 1990, 6, 186-193. [CrossRef]

6. Sun, L.Q.; Sugiyama, H.; Mizobata, K.; Minato, R.; Tojo, A. Numerical and Experimental investigations on Mach 2 and 4 Pseudo-Shock Wave in a Square Duct. Trans. Jpn. Soc. Aeronaut. Space Sci. 2004, 47, 124-130. [CrossRef] 
7. Sugiyama, H.; Tsujiguchi, Y.; Honma, T. Structure and Oscillation Phenomena of Pseudo-Shock Waves in a Straight Square Duct at Mach 2 and 4. In Proceedings of the 15th AIAA International Space Planes and Hypersonic Systems and Technologies Conference, AIAA 2008-2646, Dayton, OH, USA, 28 April-1 May 2008.

8. Lawrence, R.A. Symmetrical and Unsymmetrical Flow Separation in Supersonic Nozzles; Research Report Number 67-1; Southern Methodist University: Dallas, TX, USA, 1967.

9. Papamoschou, D.; Zill, A.; Johnson, A. Supersonic flow separation in planar nozzles. Shock Waves 2009, 19, 171-183. [CrossRef]

10. Myshenkov, E.V.; Myshenkova, E.V. Hysteresis phenomena in a plane rotatable nozzle. Fluid Mech. 2010, 45, 667-678. [CrossRef]

11. Wang, T.-S. Transient three-dimensional startup side load analysis of a regeneratively cooled nozzle. Shock Waves 2009, 19, 251-264. [CrossRef]

12. Neuendorf, R.; Wygnanski, I. On a Turbulent Wall Jet Flowing over a Circular Cylinder. J. Fluid Mech. 1999, 381, 1-25. [CrossRef]

13. Miozzi, M.; Lalli, F.; Romano, G.P. Experimental investigation of a free-surface turbulent jet with Coanda effect. Exp. Fluids 2010, 49, 341-353. [CrossRef]

14. Allery, C.; Guerin, S.; Hamdouni, A.; Sakout, A. Experimental and numerical POD study of the Coanda effect used to reduce self-sustained tones. Mech. Res. Commun. 2004, 31, 105-120. [CrossRef]

15. Wang, C.P.; Zhuo, C.F. Model for Asymmetry of Shock/Boundary Layer Interactions in Nozzle Flows. Trans. Nanjing Univ. Aeronaut. Astronaut. 2018, 35, 146-153.

16. Verma, S.B.; Manisankar, C. Origin of flow asymmetry in planar nozzles with separation. Shock Waves 2014, 24, 191-209. [CrossRef]

17. Telega, J.; Szwaba, R.; Doerffer, P. Shock Waves Asymmetry in a Symmetric Nozzle. Symmetry 2019, $11,1477$. [CrossRef]

18. Piponniau, S.; Dussauge, J.P.; Debieve, J.F.; Dupont, P. A simple model for low-frequency unsteadiness in shock induced separation. J. Fluid Mech. 2009, 629, 87-108. [CrossRef]

19. Simpson, R.L. Turbulent boundary-layer separation. Annu. Rev. Fluid Mech. 1989, 21, 205-234. [CrossRef]

20. Dupont, P.; Haddad, C.; Debieve, J.F. Space and time organization in a shock induced boundary layer. J. Fluid Mech. 2006, 559, 255-277. [CrossRef]

21. Papamoschou, D.; Roshko, A. The compressible turbulent shear layer: An experimental study. J. Fluid Mech. 1988, 197, 453-477. [CrossRef]

22. Browand, F.K.; Troutt, T.R. The turbulent mixing layer: Geometry of large vortices. J. Fluid Mech. 1985, 158, 489-509. [CrossRef]

23. Smits, A.J.; Dussauge, J.P. Turbulent Shear Layers in Supersonic Flow, 2nd ed.; AIP Press: New York, NY, USA, 2006.

(C) 2020 by the authors. Licensee MDPI, Basel, Switzerland. This article is an open access article distributed under the terms and conditions of the Creative Commons Attribution (CC BY) license (http://creativecommons.org/licenses/by/4.0/). 